\title{
MAGNETIC RECONNECTION MODELS OF PROMINENCE FORMATION
}

\author{
B. T. WELSCH \\ Space Sciences Laboratory, University of California, Berkeley, CA 94720-7450; welsch@ssl.berkeley.edu \\ C. R. DeVore \\ Laboratory for Computational Physics and Fluid Dynamics, Naval Research Laboratory, Washington, DC 20375 \\ AND \\ S. K. Antiochos \\ E. O. Hulburt Center for Space Research, Naval Research Laboratory, Washington, DC 20375 \\ Received 2005 March 9; accepted 2005 July 26
}

\begin{abstract}
To investigate the hypothesis that prominences form by magnetic reconnection between initially distinct flux systems in the solar corona, we simulate coronal magnetic field evolution when two flux systems are driven together by boundary motions. In particular, we focus on configurations similar to those in the quiescent prominence formation model of Martens \& Zwaan. We find that reconnection proceeds very weakly, if at all, in configurations driven with global shear flows (i.e., differential rotation); reconnection proceeds much more efficiently in similar configurations that are driven to collide directly, with converging motions along the neutral line that lead to flux cancellation; reconnected fields from this process can exhibit sheared, dipped field lines along the neutral line, consistent with prominence observations. Our field configurations do not possess the "breakout" topology, and eruptions are not observed, even though a substantial amount of flux is canceled in some runs.
\end{abstract}

Subject headings: MHD — Sun: activity — Sun: magnetic fields — Sun: prominences

\section{INTRODUCTION}

Prominences are long $\left(\sim 10^{2} \mathrm{Mm}\right)$, thin $(\sim 3 \mathrm{Mm})$, relatively cold $(\lesssim 0.05 \mathrm{MK})$ density enhancements in the low solar corona that form striking extended emission structures over the solar limb in chromospheric spectral lines (e.g., $\mathrm{H} \alpha$ ). When viewed in similar lines on the disk, these structures form sinuous absorption features, known as filaments. The sudden eruption of prominences (sometimes occurring over a few tens of Alfvén crossing times) can lead to coronal mass ejections, or CMEs (Forbes 2000). As CMEs are the primary drivers of severe space weather disturbances (Gosling 1993), solar physicists are intently studying the origin, structure, evolution, and eruption of prominences, all of which remain poorly understood.

The magnetic fields that thread prominences can sustain their masses against gravity via magnetic tension in concave-up (dipped) field lines (Kippenhahn \& Schlüter 1957; Kuperus \& Raadu 1974). In addition, Karpen et al. (2001) have shown that heating can drive formation of density enhancements in long, low-lying field lines that range from dipped to weakly arched. These mechanisms permit prominences to exist for days or weeks, much longer than the minutes-long coronal free fall time of their constituent density enhancements, while changing only slowly. Magnetic fields also keep the cool, dense prominence material thermally isolated from the surrounding hot, tenuous corona.

In this work, we concentrate on processes that generate prominence-like magnetic field configurations. The essential content of most prominence formation models is their explanation of how dipped field lines arise in the corona. Many mechanisms have been advanced, including emergence of twisted flux ropes through the photosphere (e.g., Rust \& Kumar 1994); photospheric twisting of emerged bipolar fields (e.g., Priest et al. 1989); localized shearing of coronal field lines' footpoints along the polarity inversion line (PIL) in an emerged bipolar flux system (Antiochos et al. 1994; DeVore \& Antiochos 2000); and photo- spheric convergence and cancellation, with coronal reconnection, between emerged flux systems (e.g., van Ballegooijen \& Martens 1989; Martens \& Zwaan 2001).

In addition to explaining how dipped field lines arise, formation models must also match other observed properties of prominences (see Démoulin [1998] and Martin [1998] for comprehensive reviews). Some polarity inversion lines (PILs) of the photospheric magnetic field's normal component, known as filament channels, notably lack chromospheric $\mathrm{H} \alpha$ fibrils crossing the PIL, but possess fibrils with axes parallel to the PIL, indicating that the horizontal magnetic field also parallels the PIL. While these channels are observed to exist independently of the presence of prominence material, prominences only form above and along PILs in such channels. A majority of prominences (about 65\%) lie along PILs between two active regions (Tang 1987). Within prominences, magnetic field components perpendicular to their axes most often (in $\gtrsim 90 \%$ of cases) point in the "inverse" sense, from the negative sides of their photospheric PILs toward the positive sides (Bommier \& Leroy 1998). Above the filament channel (and the prominence, if present), field lines, visible as X-ray and EUV arcades, link the photospheric flux distributions across the PIL, from positive to negative (Martin \& McAllister 1996); the angle between these overlying field lines and the channel's axis increases with increasing height above the channel, from nearly parallel toward perpendicular.

Martin (1998) characterized the convergence of opposing magnetic flux toward the PIL, followed by cancellation, as a "necessary" condition for the formation of prominences. The observed convergence of magnetic flux toward a PIL may be only a phase effect as the U-shaped field lines in the bottom half of a horizontal flux rope emerge. This would suggest that fields on either side of the PIL should be concave upward prior to prominence formation, which accords with recent observations of PILs within active regions by Lites (2005). Cancellation along PILs between active regions that emerged separately can also be explained 
by the emergence of a flux rope, if the rope links the two beneath the photosphere (see van Driel-Gesztelyi et al. [2000] for a possible observation); the axis of such a flux rope would lie at $90^{\circ}$ to the PIL.

Observed relationships between various aspects of prominences' magnetic environments led to a definition of chirality, or handedness, for them: when viewed from the positive-flux side of the PIL, the axial field in a dextral filament points to the right, while the field in a sinistral filament channel points to the left (Martin et al. 1994). The sense of rotation of field direction with increasing altitude is left-skewed with respect to the field along the channel axis in dextral channels' arcades (consistent with negative magnetic helicity), and is right-skewed in sinistral channels (consistent with positive magnetic helicity), as described by Martin \& McAllister (1996). The prominences' chirality shows a hemispheric preference: statistically, negative-helicity/left-skewed/dextral prominences predominate in the northern hemisphere, and positivehelicity/right-skewed/sinistral prominences in the southern (Zirker et al. 1997).

In a survey of filament chiralities, Pevtsov et al. (2003) found the latitudinal chirality distribution function to be flat on either side of the equator, with a sharp gradient across the equator. They suggested that chirality due to Coriolis effects would cause the distribution to have extrema toward the poles, while chirality from either differential rotation or the subphotospheric dynamo would cause extrema at midlatitudes (in the region of maximum differential shear and in the activity belt, respectively). They explained the observed distribution in terms of the accumulation of magnetic helicity in filaments and filament channels via magnetic reconnection, which they suggested would smooth out any localized extrema in the chirality source distribution. They noted that this model also explains why the hemispheric preference in relatively long-lived quiescent filament chiralities is stronger than the hemispheric preferences in either shorter-lived active region filament chiralities or active region current helicities (Pevtsov 2002).

Antiochos et al. (1994) and DeVore \& Antiochos (2000) have presented "sheared arcade" models that exhibit many of the observed properties of prominences, most notably sheared field lines with dips and the inverse configuration. Photospheric shear flows along PILs, similar to those used to generate these model configurations, have been observed (e.g., Welsch et al. 2004), although the observed flows lack the duration of the model flows.

The quiescent prominence formation model of Martens \& Zwaan (2001) explains the development of prominences in terms of photospheric convergence and cancellation between flux systems from different bipolar active regions. The essence of the model is that bringing the regions' photospheric flux distributions together - whether by shear from differential rotation, converging motion, or diffusive random walk - drives reconnection between the flux systems. The reconnected flux forms the body of the prominence, as well as the overlying arcade.

Galsgaard \& Longbottom (1999) used a magnetohydrodynamics (MHD) code to test a similar idea in a configuration in which two bipolar photospheric flux distributions that were initially magnetically connected in the model corona were driven toward each other by imposed converging motions at the model photosphere. The driving induced strong currents between the flux systems, and the ensuing reconnection increased their shared flux. Some dipped and helical reconnected field lines formed and supported density enhancements over the PIL, consistent with prominence observations. More recently, Mackay and collaborators (Mackay \& van Ballegooijen 2005; Mackay \& Gaizauskas 2003; Mackay \& van Ballegooijen 2001) have used magnetofrictional codes with diffusive flux transport models to study the formation of filaments that formed via cancellation between model active regions.

In a series of numerical MHD experiments, we have investigated the Martens \& Zwaan (2001) model from an initial condition in which the two flux distributions are not initially linked in the model corona, consistent with the emergence of distinct active-region flux systems. We impose either a global shear flow, representing differential rotation, or flows that converge toward the PIL, which drive a cancellation of photospheric flux. In addition, we model the effect of preexisting twist in the initial field by rotating a portion of the active-region flux prior to imposing the shearing or converging flows. In $\S 2$, we discuss the numerical model. In $\S 3$, we describe our experiments using shearing flows or converging flows to drive the bottom boundary, and present our results for each case. In $\S 4$, we discuss the implications of these results.

\section{MODEL}

To model the coronal response to a prescribed photospheric evolution, we used the adaptively refined MHD solver (ARMS, C.R. DeVore et al. 2005, in preparation) with a Cartesian simulation volume. ARMS solves the following ideal MHD equations:

$$
\begin{gathered}
\frac{\partial \rho}{\partial t}+\nabla \cdot(\rho \boldsymbol{v})=0 \\
\frac{\partial U}{\partial t}+\nabla \cdot(U \boldsymbol{v})+P \nabla \cdot \boldsymbol{v}=0 \\
\frac{\partial(\rho \boldsymbol{v})}{\partial t}+\nabla \cdot(\rho \boldsymbol{v} \boldsymbol{v})+\nabla P-\frac{1}{\mu}(\nabla \times \boldsymbol{B}) \times \boldsymbol{B}=0 \\
\frac{\partial \boldsymbol{B}}{\partial t}-\nabla \times(\boldsymbol{v} \times \boldsymbol{B})=0,
\end{gathered}
$$

where $t$ denotes time, $\boldsymbol{v}$ the velocity field, $\boldsymbol{B}$ the magnetic field, $\rho$ the mass density, $U$ the internal energy density, $P$ the plasma pressure, and $\mu$ the magnetic permeability. The internal energy density and pressure are related via $U=P /(\gamma-1)$, where $\gamma$ is the ratio of specific heats. We set $\gamma$ and $\mu$ to $5 / 3$ and $4 \pi$, respectively, and the gas constant to the scaled value $R / m=0.005$.

We focus on the evolution of the magnetic structure in our simulated corona, and choose a simplified treatment of the thermodynamics of the low- $\beta$ plasma in our model. Accordingly, we ignore the effects of coronal heating, thermal conduction, and radiation. We begin our runs with a uniform initial mass density and temperature. Scaled initial values of $\rho=2$ and $T=1$ were chosen to give an initial gas pressure $P=0.01$ and sound speed $c_{s}=0.09$.

Extrapolative, zero-gradient, open boundary conditions were used for all the variables on all sides of the computational box, with the exception of the bottom. The bottom boundary is closed, with the normal velocity constrained to vanish and the tangential velocity prescribed as detailed in $\S 3$.

The ARMS simulation code is a time-explicit, conservative, monotone MHD model whose equations are advanced using flux-corrected transport techniques (DeVore 1991). Numerical diffusion inherent in the algorithm allows magnetic reconnection to occur even though the equations solved are nominally ideal. The adaptive mesh refinement is managed by the PARAMESH toolkit (MacNeice et al. 2000). The grid is refined adaptively based upon the spatial variations of magnetic field strength and electric current density. Linear interpolation with a monotone limiter was used to initialize newly created grid points. For numerical convenience, we ran our experiments using just two levels of 
refinement. As discussed below, we believe that additional grid refinement would not significantly alter our results.

\section{EXPERIMENTS}

The control variables in our simulations are the initial flux distribution on the bottom boundary, $B_{z}(x, y, 0 ; 0)$, and the velocity field at the bottom boundary, $\boldsymbol{v}(x, y, 0 ; t)$, that we use to drive the coronal system.

In our experiments, the initial field at $\boldsymbol{x}=(x, y, z)$ in the Cartesian computational domain was a superposition of a constant horizontal background field, $\boldsymbol{B}_{0}$, and the field from a collection of point dipole sources,

$$
\boldsymbol{B}^{(\mathrm{TOT})}(\boldsymbol{x})=\boldsymbol{B}_{0}+\sum_{i=1}^{N} \frac{3\left[\boldsymbol{m}_{i} \cdot\left(\boldsymbol{x}-\boldsymbol{x}_{i}\right)\right]\left(\boldsymbol{x}-\boldsymbol{x}_{i}\right)-\boldsymbol{m}_{i}\left|\boldsymbol{x}-\boldsymbol{x}_{i}\right|^{2}}{\left|\boldsymbol{x}-\boldsymbol{x}_{i}\right|^{5}},
$$

where $\boldsymbol{m}_{i}$ is the dipole moment of the $i$ th source, and $\boldsymbol{x}_{i}$ its location (restricted to lie outside the computational domain). The dipoles' moments and locations were chosen such that both the initial peak Alfvén speed and the characteristic length scale were near unity. The constant background field served two purposes: it kept the "interesting" field evolution low in the computational volume, thereby reducing the vertical extent of the domain that needed to be simulated; and it prevented the Alfvén speed from becoming too small in the upper zones of the box (where the magnetic field is quite weak, but the density remains high), thereby reducing the time necessary for the configuration to relax after driving ceased. The ratio of the peak field strength on the bottom boundary to the background field strength (set to 0.1 in all runs) was always greater than 30:1.

Each of the two bipolar "active region" flux distributions was composed of three dipoles: two oriented vertically (one upward, one downward), and one horizontally (pointing toward the upward dipole). This arrangement forms two concentrated regions of magnetic flux, as opposed to the relatively diffuse flux regions that result from using a single horizontal dipole.

We evolve the system through separate driving and relaxation phases. During the driving phases, boundary velocities between $1 \%$ and $7 \%$ of the simulation's maximum Alfvén speed were used. While even these highly sub-Alfvénic driving speeds are unrealistically fast for the Sun, they permit simulations that consume substantially less computer time. Further, extremely slow driving is unnecessary, as we are ultimately concerned with the coronal field's evolution on timescales that are long compared to the ideal MHD timescale, which is of order unity in our simulations. The driving boundary velocities were modulated with a time profile

$$
g(t)=1-\cos \left(2 \pi t / \tau+\phi_{t}\right)
$$

that smoothly turned the flows on and off; both $\boldsymbol{v}$ and $\partial \boldsymbol{v} / \partial t$ vanished at the profiles' endpoints. Typically, $\tau=60$ was used. After each driving phase, the field was allowed to relax for hundreds of photospheric Alfvén times (typically 200), until significant flows ceased.

The nature of the imposed flows - shearing or convergingnaturally divides the runs into two categories. Each category may be further divided based on the field configuration prior to shearing or converging: either the initial field was potential (currentfree), or we had previously "spun up" initially potential fields to impart twist. This spin-up was used to model active region flux systems that have been observed to emerge carrying current (Leka
TABLE 1

\begin{tabular}{|c|c|c|}
\hline Dipole Moment & $\begin{array}{c}\text { Location } \\
(x, y, z)\end{array}$ & $\begin{array}{l}\text { Orientation } \\
\left(n_{x}, n_{y}, n_{z}\right)\end{array}$ \\
\hline \multicolumn{3}{|c|}{ Run A } \\
\hline $0.5 \ldots \ldots \ldots \ldots \ldots \ldots \ldots$ & $(+1.58,+0.32,-0.50)$ & $(0.00,0.00,1.00)$ \\
\hline $0.5 \ldots \ldots \ldots \ldots \ldots \ldots . . .$. & $(-1.20,+1.07,-0.50)$ & $(0.00,0.00,-1.00)$ \\
\hline 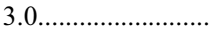 & $(+0.19,+0.69,-2.12)$ & $(0.97,-0.26,0.00)$ \\
\hline $0.5 \ldots \ldots \ldots \ldots \ldots \ldots$ & $(+1.20,-1.07,-0.50)$ & $(0.00,0.00,1.00)$ \\
\hline $0.5 \ldots \ldots \ldots \ldots \ldots \ldots$ & $(-1.58,-0.32,-0.50)$ & $(0.00,0.00,-1.00)$ \\
\hline $3.0 \ldots \ldots \ldots \ldots \ldots \ldots \ldots \ldots$ & $(-0.19,-0.69,-2.12)$ & $(0.97,-0.26,0.00)$ \\
\hline \multicolumn{3}{|c|}{ Run B } \\
\hline $0.5 \ldots \ldots \ldots \ldots . . . . .$. & $(0.00,-2.00,-0.50)$ & $(0.00,0.00,-1.00)$ \\
\hline $0.5 \ldots \ldots$ & $(+3.00,-2.00,-0.50)$ & $(0.00,0.00,+1.00)$ \\
\hline $9.0 \ldots \ldots \ldots \ldots \ldots \ldots . .$. & $(+1.50,-2.00-3.00)$ & $(+1.00,0.00,0.00)$ \\
\hline $0.5 \ldots \ldots \ldots$ & $(-1.00,+2.00,-0.50)$ & $(0.00,0.00,+1.00)$ \\
\hline 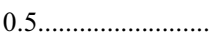 & $(-3.00,+2.00,-0.50)$ & $(0.00,0.00,-1.00)$ \\
\hline 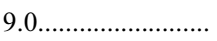 & $(-1.50,+2.00-3.00)$ & $(+1.00,0.00,0.00)$ \\
\hline
\end{tabular}

Simulation Parameters

et al. 1996). After relaxation, these fields are approximately forcefree in our low-pressure corona. The spin-up was accomplished by driving with incompressible velocities tangent to contours of normal $(\hat{z})$ flux,

$$
\boldsymbol{v}_{\perp}=f_{ \pm}\left(B_{n}\right)\left(\hat{n} \times \nabla B_{n}\right),
$$

where $f_{ \pm}\left(B_{n}\right) \equiv \mp(.0075) \sin \left(\pi b_{n} / 2\right)$, with

$$
b_{n} \equiv \begin{cases}0 & \text { for }\left|B_{n}\right| \leq B_{-}, \\ \left(\left|B_{n}\right|-B_{-}\right) /\left(B_{+}-B_{-}\right) & \text {for } B_{-} \leq\left|B_{n}\right| \leq B_{+} \\ 1 & \text { for } B_{+} \leq\left|B_{n}\right|\end{cases}
$$

where $f_{+}\left(f_{-}\right)$is chosen for positive (negative) flux distributions, and $B_{-}=3$ and $B_{+}=4$. These flows spin up strong-field regions while leaving weak-field regions unaltered. The sense of rotation of both flux distributions is counterclockwise, which injects negative helicity into the corona, consistent with observations of active region fields in the northern hemisphere (Pevtsov et al. 1995). As implemented, the driving injected about half a turn in the most twisted field lines.

This is probably appropriate for the solar case, where the twist per unit length, $\sim 10^{-8} \mathrm{~m}^{-1}$, inferred from both vector magnetograms (Pevtsov et al. 1995) and quasi-empirical prominence models (Aulanier et al. 2002), implies that $100 \mathrm{Mm}$ field lines are twisted about one turn or less.

\subsection{Driving With Shear}

In our shearing runs, we drove the bottom boundary of the box with a linear shear profile,

$$
\boldsymbol{v}=-\Omega_{0} y \hat{x}
$$

which models the shear from differential rotation in the northern hemisphere, where $\hat{y}$ points north, and $\hat{x}$ points to the west limb of the Sun in the plane of the sky. For typical shearing runs, $\Omega_{0}=0.01$ was used. Here, we focus on results from one shearing run in particular, which we label run A.

The locations, strengths, and orientations of the dipoles initializing run $\mathrm{A}$ are listed in Table 1 . This run was initialized on a coarse grid of $N_{x} \times N_{y} \times N_{z}=96 \times 64 \times 64$ grid points. The fieldstrength refinement criterion led to an immediate increase of 

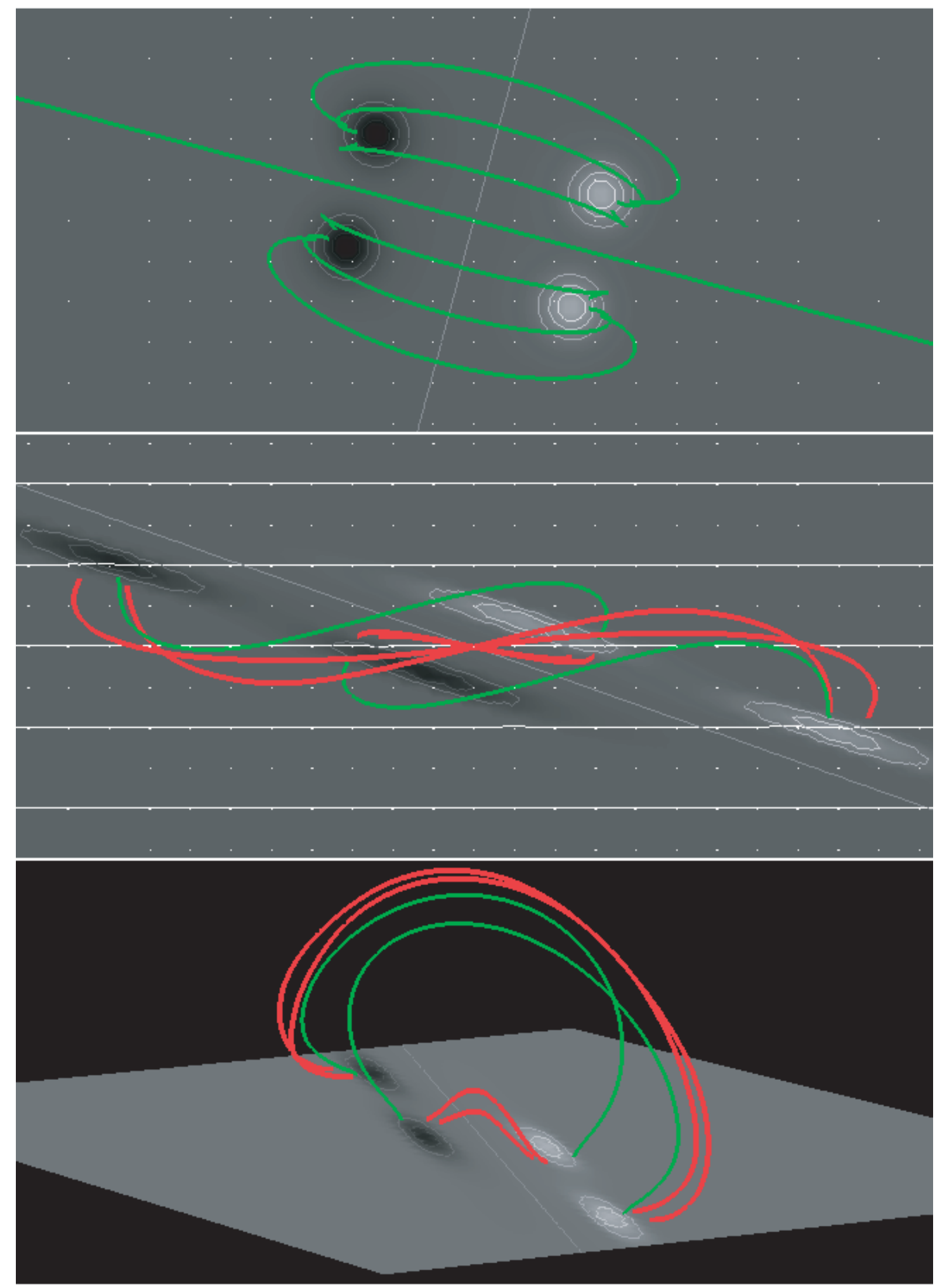

FIG. 1.-Top: Starting from an initial condition with two bipolar flux systems that, by symmetry, are not magnetically linked in the corona, we drove the bottom boundary with a global, linear shear profile to model differential rotation. Middle: White lines are contours of $v_{x} \propto-\Omega_{0} y$. Even after prolonged shearing has brought two initially unconnected strong-flux regions into close proximity, very little flux has reconnected to link the two. Bottom: Typical examples of reconnected field lines are arched, and lack the flat, dipped, or helical structure expected in prominence fields. 


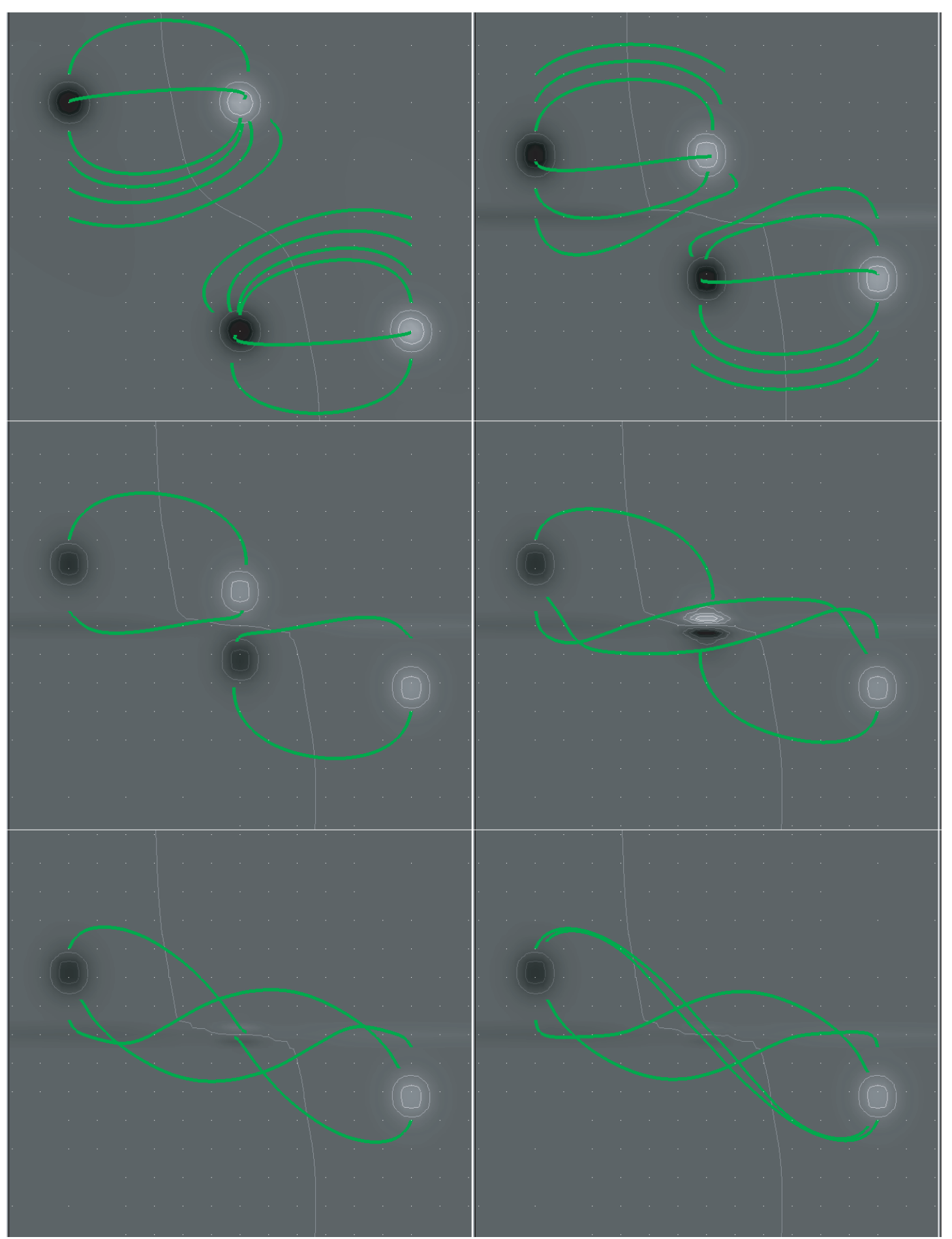

FIG. 2.-Top left: Sample field lines from the initially potential state. Top right: Field lines with the same outer footpoints, after the first convergence phase. Middle left: During the second convergence phase, only the inner two polarities converged. Middle right: Flux pileup can been seen at the convergence site between the cancelling fluxes. Bottom left: Two field lines immediately prior to cancellation-induced reconnection. Bottom right: Field lines from the same two footpoints immediately afterward. 

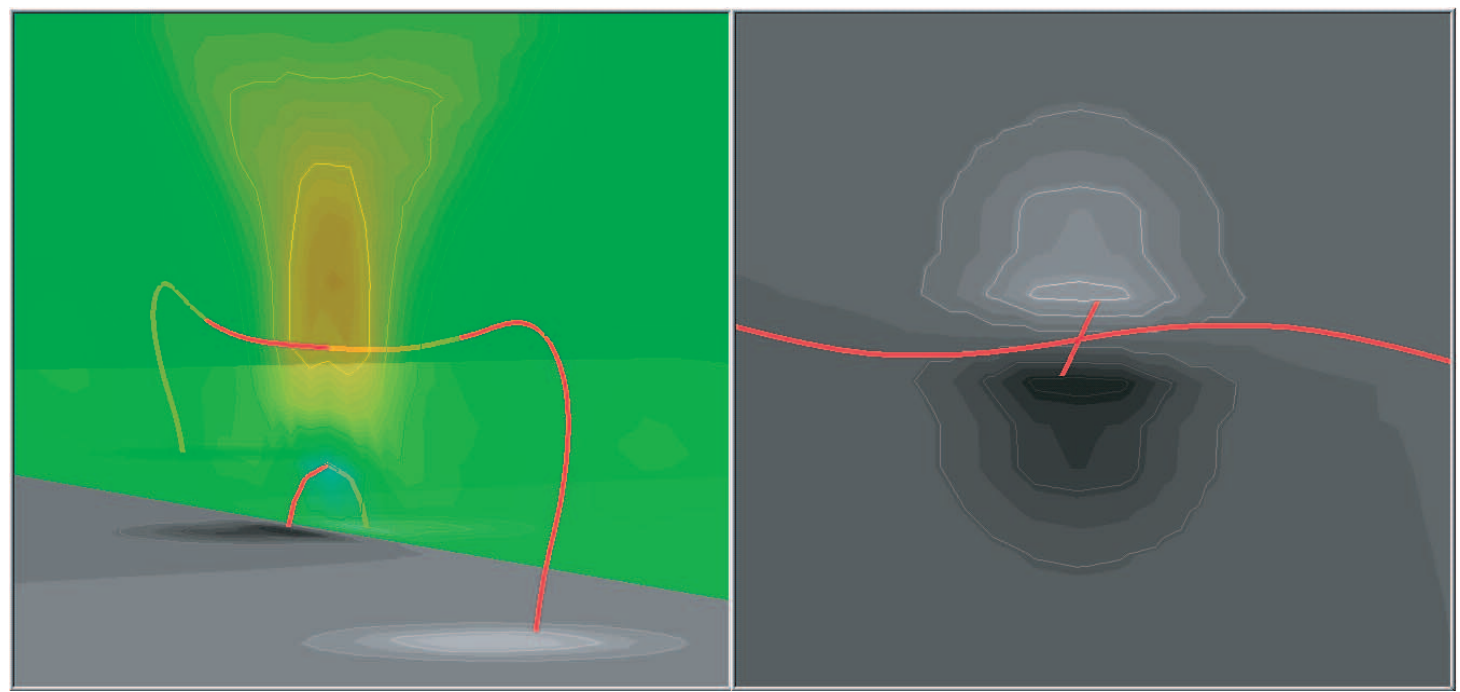

FIG. 3.-Perspective (left) and overhead (right) views of a pair of post-reconnection field lines. Color-filled contours of vertical velocity, $v_{z}$, are shown in cross section (left; red is upward, blue is downward). The higher line is moving up while the lower line is moving down, both directed away from the region between them where they evidently reconnected. In this image, peak vertical velocities are less than $20 \%$ of the total Alfvén speed.

about $55 \%$ in the number of grid points; the code refined the grid further during the run, reaching a maximum increase of $175 \%$. These changes are much smaller than the $700 \%$ increase required to refine the grid by a factor of 2 over the entire domain. One root block's lateral spatial extent should correspond, roughly, to a few tens of megameters on the Sun. Our initial field configuration is illustrated in the top panel of Figure 1. The active regions' flux distributions possess a northern hemisphere Joy's law tilt (Hale et al. 1919) of $15^{\circ}$ with respect to the $x$ direction. A uniform background field parallels the initial axes of the bipoles. The peak initial Alfvén speed was 0.70 .

The results of run A are typical of several global shearing runs that we conducted. These experiments assumed both potential and spun-up initial states, and both finite and zero Joy's law tilts. Quite generally, we find that (1) very little flux reconnects, even after the flux distributions are very strongly sheared (e.g., Fig. 1, middle panel); and (2) the reconnected field lines are rather strongly arched, not dipped, flat, or helical (Fig. 1, bottom panel). The moderate twist in our spun-up initial fields was insufficient to produce any dips or helices in the reconnected flux. In passing, we note that this configuration possesses sigmoidal field lines of both right- and left-handedness when viewed from above- - e.g., the longer and shorter red field lines.

The lack of appreciable reconnection evidently reflects the relatively simple topological structure of the field in our simulations: no nulls or separatrices are present in the simulation volume, across which strong current sheets can develop and promote fast reconnection. Furthermore, the smooth, global structure of the driving flows implies that regions with large spatial gradients in footpoint connectivities-quasi-separatrix layers (Priest 1985), the expected sites of reconnection without nulls - develop at most linearly in time, and are smoothed out with minimal reconnection. In contrast, configurations with nulls and the attendant separatrix surfaces have discontinuities in field-line connectivity that cannot be dissipated away.

\subsection{Driving with Convergence}

In a second approach, we drove the bottom boundary such that one pair of opposite-polarity flux distributions (one polarity from each bipolar active region) converged and eventually canceled, starting from both potential and spun-up initial field configurations. As the resulting field in this and similar runs possessed many characteristics of prominence fields, we describe run $\mathrm{B}$ and its results in much greater detail than run A. The dipoles' locations, strengths, and orientations for this run are shown in Table 1. This run was initialized with $N_{x} \times N_{y} \times N_{z}=128 \times 96 \times 32$ root blocks, with initial and peak refinements increasing the number of points by $45 \%$ and $185 \%$. The peak initial Alfvén speed in this run was 0.66 , and the background field in this case ran in the $-\hat{x}$ direction.

The initial normal-field boundary condition, shown in the top left panel of Figure 2, was driven with a large-scale compressible convergence flow,

$$
v_{y}(y)= \begin{cases}-v_{0} \cos (\pi y / 12) & \text { for } 0<y<6 \\ +v_{0} \cos (\pi y / 12) & \text { for }-6<y<0\end{cases}
$$

where $v_{0}=0.01$, and the latitudinal extent of the computational domain is $y \in(-6,6)$. The convergence was implemented in two phases, the first of which encompassed the entire longitudinal extent of the computational domain, $x \in(-8,8)$. This brought the two bipolar flux systems closer together, as shown in the top right panel of Figure 2, after which the configuration was allowed to relax. The resulting coronal topology was highly nonpotential, with a current sheet separating the neighboring flux systems. This configuration models the coronal field after two magnetically isolated bipolar active regions in close proximity emerge into a preexisting field. That the two flux systems remain essentially distinct and disconnected after this first driving phase indicates that minimal reconnection occurred, even though quasi-separatrix layers were present. Field lines in both of the top panels of Figure 2 were integrated from the same footpoints in the model photosphere, backward from each panel's left side, and forward from each panel's right.

The second episode of driving led to the convergence of neighboring opposite polarities from the two bipolar systems, and encompassed a narrow longitudinal interval, $x \in(-0.75,0.75)$. As the polarities converged, the photospheric fluxes came into contact and began to cancel. This caused field lines in the overlying coronal flux systems to reconnect, creating new connections between the remote polarities of the formerly distinct bipolar regions. 

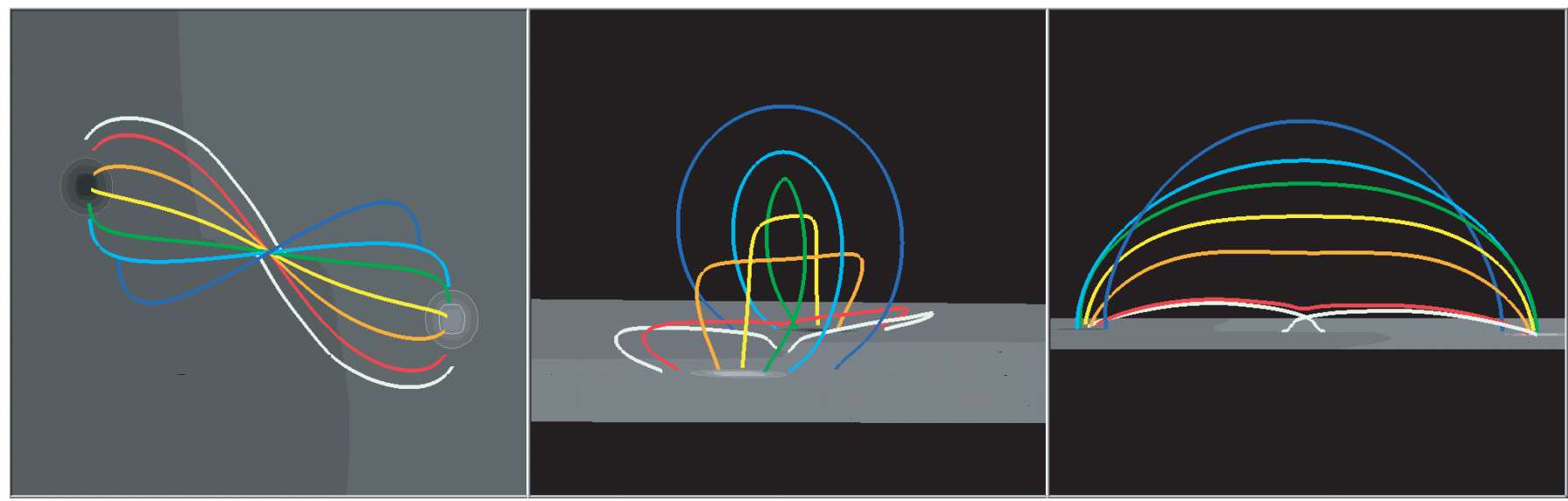

FIG. 4.-Overhead (left), end-on (middle), and edge-on (right) views of post-convergence field lines, formed by convergence and cancellation in initially potential fields. All field lines shown have reconnected, except the white pair. Note the flat or slightly dipped field lines, expected sites of plasma condensation to form prominences.

Pre-reconnection field lines, like the two nearly horizontal lines in the left center panel of Figure 2, give way to post-reconnection field lines like the two far longer S-shaped field lines in the right center panel, and also to short loops connecting the colliding polarities (not shown in Fig. 2, but see Fig. 3). The four field lines shown in each of the bottom four panels of Figure 2 were integrated from the same four points on the model photosphere, two on each side.

The cancellation and reconnection resulted from numerical diffusion at the grid scale, as the oppositely-directed flux systems were compressed against each other by the converging flows for $|y|>0$, and diffused across the $y=0$ plane.

In Figure 3, we show differing views of a pair of postreconnection field lines, in the midst of the cancellation phase. To illustrate the outflow velocities, we show contours of vertical velocity, $v_{z}$, in the $y=0$ plane in the left panel; peak vertical velocities in this image are less than $20 \%$ of the Alfvén speed. We discuss the solar analog of this process in more detail in $\S 4$.

The flux cancellation at the model photosphere and magnetic reconnection in the model corona continue as the converging flows persist in driving the opposite-polarity fluxes together (Fig. 2, bottom panels). At this stage, pre-reconnection field lines, such as the pair whose ends nearly touch in the middle of the lower left panel, produce Z-shaped post-reconnection field lines, like the two shown connecting the remote polarities in the lower right panel, together with (omitted) short loops connecting the cancelling polarities. We note that the reconnection generates both S- and Z-shaped sigmoidal field lines. This might be a fairly common feature of nonpotential magnetic fields, as it has been found in other numerical experiments (see, e.g., the upper panels of Fig. 3 in Magara \& Longcope 2001).

Finally, after the inner polarities almost completely canceled, the driving was turned off and the system was allowed to relax, resulting in the configuration seen in Figure 4. As may be seen, the lowest lying reconnected field lines (the white lines are the only field lines shown that were not formed by reconnection) are flat or slightly dipped. The low lines cross the PIL (which runs, roughly, from upper left to lower right) from negative to positive, in the inverse sense. In contrast, higher field lines are arched, and cross the PIL in the normal sense. Hence, the lowest field lines appear highly sheared, while the overlying field lines become progressively more normal to the PIL with height, consistent with filament observations. We expect that a similar state would result if the inner polarities had not canceled completely: the most recently reconnected field lines would still be flatter, and more nearly parallel to the neutral line, than the overlying, previously reconnected field lines.

For this run, the kinetic and magnetic energies, as functions of time, are shown in Figure 5. The convergence (relaxation) phases are labeled $\mathrm{C} 1$ and $\mathrm{C} 2$ (R1 and R2), with arrows denoting the extent of each interval. Several noteworthy features, labeled F1-F5, are present in the plots. First, local maxima in the magnetic and kinetic energies occur during the convergence phases, near F1 and F3, and arise from the driving. Another local maximum in the magnetic energy, marked as F2 at $t=290$, occurs well into the first relaxation phase, and results from the convergence of the two flux systems in the model corona, which lags behind the convergence on the $z=0$ plane. This lag arises because, with uniform mass density, the rapid decrease in field strength with height causes an attendant decrease in the local Alfvén speed with height.

The rapid drop in magnetic energy between F3 and F4 coincided with the onset of photospheric cancellation, implying that cancellation can rapidly decrease magnetic energy in the corona. The sudden flattening of the magnetic energy curve between F4, at $t=880$, and $\mathrm{F} 5$, at $t=1100$, probably arises from a superposition of continuing (but diminished) energy loss as the flux

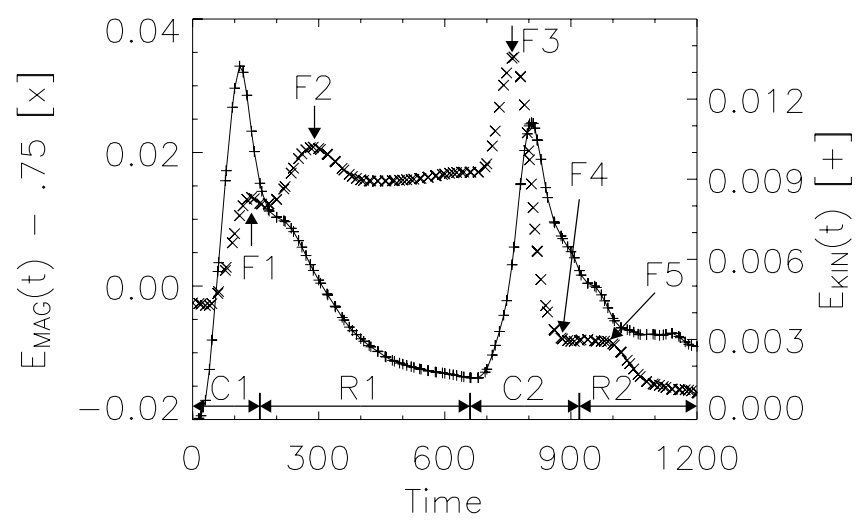

FIG. 5.-Deviation in magnetic energy $(\times)$ from its average value of 0.75 , and kinetic energy $(+)$ for the initially potential version of run $\mathrm{B}$. The convergence (relaxation) phases are labeled $\mathrm{C} 1$ and $\mathrm{C} 2$ (R1 and $\mathrm{R} 2$ ); arrows denote the temporal extent of each interval. Several interesting features in these plots, denoted F1-F4, are described in the text. 

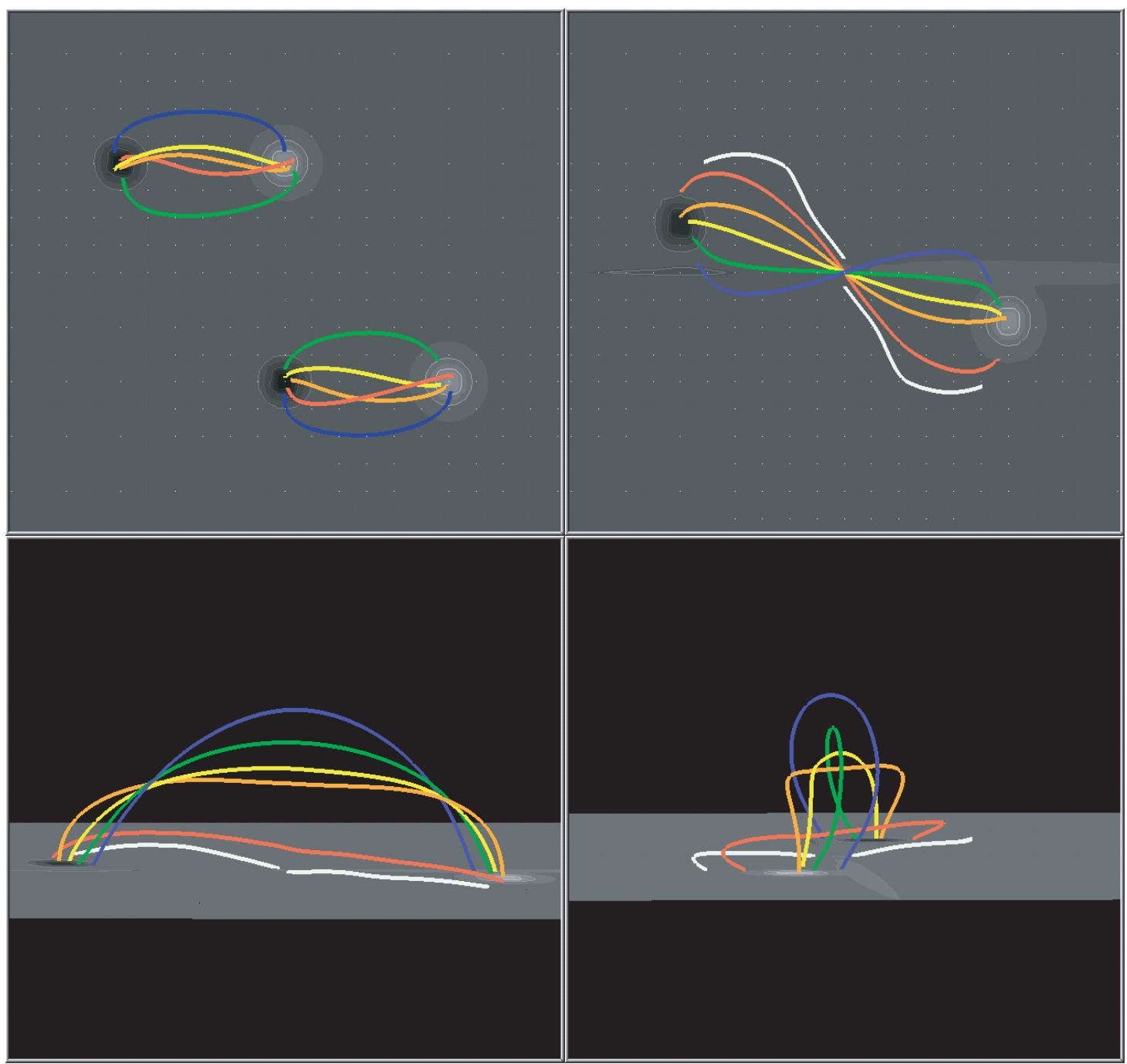

Fig. 6.-Perspective (top left), overhead (top right), edge-on (bottom left), and end-on (bottom right) views of post-cancellation, reconnected field lines, for converging spun-up fields.

cancellation ceases, and a temporary increase in coronal magnetic energy, similar to the local maximum near F2.

In Figure 6, we show results from a run in which the fields were spun up prior to convergence and cancellation. As in the previous case, many field lines in the final configuration are flat or weakly dipped. Also as in the previous case, the field runs essentially parallel to the final PIL, and crosses the PIL from negative to positive, in the inverse sense. Further, in both cases the direction of the reconnected field rotates from parallel to perpendicular to the PIL with increasing altitude.

\section{DISCUSSION}

\subsection{Photospheric Driving and Reconnection}

Martens \& Zwaan (2001) presented a model which predicts that shearing and convergence/cancellation lead to magnetic reconnection between initially separate active regions, with subsequent prominence formation. We simulated these processes by applying either global-scale shear flows or converging flows to the footpoints of field lines in model active regions. We found that shearing drove reconnection much less efficiently than convergence with cancellation did, for footpoint displacements of similar spatial extent. Sampling the field lines in the region of interaction between the active regions revealed many more link- ing field lines in the cases with convergence and cancellation than in those with shear only.

We attribute the differing amounts of reconnection to the profound difference between the cases of shearing (or twisting) flows versus converging flows that lead to cancellation. The former produce currents on scales similar to those of the imposed flows, the shear scale; if that scale is global, as for differential rotation, the current scale will be global, too. This leads to distributed volume currents, which can be maintained for a very long time in the real corona and in our simulations, and cause minimal reconnection. The latter must eventually produce current sheets that develop on the local dissipation scale: the resistive scale length in the corona, and the grid scale in our simulations. These current sheets allow the magnetic field to reconnect efficiently, as we have seen in our simulations.

Aside from cancellation, however, we found that neither shearing nor converging motions led to much reconnection. This suggests that, without nulls or separatrices, flux transport on the photosphere does not produce significant reconnection.

In the convergence and cancellation scenario, run B, we found that convergence of footpoints from differing flux systems led to both magnetic reconnection between the flux systems in the model corona, and to cancellation of flux at the model photosphere. In our simulations, both reconnection and cancellation 
result from numerical diffusion. (Increasing the grid resolution in this case would lower this diffusivity, but the converging flows would still force reconnection and cancellation eventually.) Analogous field evolution might occur on the Sun. From observations of flux convergence and cancellation, Chae et al. (2002) and Chae (2003) suggest that, as opposite-polarity photospheric flux distributions approach each other, a current sheet can develop between their overlying flux systems, leading to magnetic reconnection above the converging features. Subsequently, the newly created short field lines that link the cancelling fluxes can submerge, as suggested by observations reported by Harvey et al. (1999) and Chae et al. (2004), while the newly created, longer field lines move upward into the corona. In fact, Chae et al. (2001, 2003) report an example of prominence formation in NOAA AR 8668 that resembles the scenario presented here in many details.

While Martens \& Zwaan (2001) modeled the combined action of shearing and convergence, we did not model the two processes simultaneously. As a practical matter, properly timing the collision of flux distributions shearing past each other is not trivial. On the Sun, however, flux collisions are systematic: turbulent photospheric flows disperse active region fields and drive the resulting small patches of flux toward downflow regions, where they encounter like- or opposite-sign flux; in the latter case, cancellation results. While it is reasonable to try to combine global shearing and convergence in a future study, we have, in fact, taken an easier first approach to this problem. By spinning up the active region fields prior to converging them in one of our runs, we tested the effects of volumetric currents introduced by twist rather than shear on the convergence and cancellation process. Results from our twisted and untwisted runs did not differ appreciably.

In both of our runs, the initially unconnected active region fields are assumed to have emerged separately, before the runs began. Hence, the photospheric field along the PIL between our model active regions would not be concave upward. Our simulations, therefore, are not consistent with recent observations by Lites (2005) that show that fields along PILs within active regions tend to be concave upward.

\subsection{Prominence-like Fields}

In addition, we found that, while the weak reconnection induced by global shearing did not produce prominence-like field lines, reconnection induced by convergence and cancellation did yield such configurations: some reconnected field lines were flat or weakly dipped; the field exhibited a parallel-to-perpendicular structure with increasing height above the PIL; and the field possessed an inverse configuration seen in most prominences. Convergence and cancellation acting on nonpotential, spun-up fields led to similar configurations. As the initial photospheric flux distribution in this run corresponds to the expected situation on the Sun's northern hemisphere (Martens \& Zwaan 2001), and the resulting field possessed negative helicity, left-skewed overlying field lines, and a dextral chirality, this model obeys the hemispheric chirality rules we reviewed in $\S 1$.

The agreement between these simulations and observations suggests that convergence and cancellation processes similar to those modeled here are viable mechanisms for prominence formation. The success of convergence and cancellation accords with Martin's (1998) assertion that converging flows are a necessary condition for the formation of a prominence.

\subsection{Increase in Coronal Helicity}

Interestingly, the sequence of footpoint cancellations and subsequent reconnections in run B leads to nonzero twist in post-reconnection fields, even in the initially potential run. This post-reconnection twist, and its associated magnetic helicity, is a manifestation of the relative helicity (Berger \& Field 1984) imparted by the converging flows. The resulting head-to-tail linkage leads to a buildup of net twist in the reconnected field, though not to an enhancement of twist density: for identical twist densities in the pre-reconnection flux systems, the twist per unit length is nearly unchanged, while the total twist in the reconnected domain approximately doubles as the field lines' lengths double.

Even though our post-cancellation flux system does possess some twist, our prominences more closely resemble sheared arcade models (Antiochos et al. 1994; DeVore \& Antiochos 2000) than the highly-twisted flux rope in flux cancellation models described by Amari et al. (1999), Linker et al. (2001), and Lionello et al. (2002). Also unlike these models, we did not impose either an enhanced magnetic diffusivity or a noninductive electric field at the simulation's bottom boundary to achieve flux cancellation during run $\mathrm{B}$.

\subsection{Increase in Coronal Free Energy}

To study the change in the free magnetic energy in the simulation volume due to the flux cancellation, we evaluated the energy in potential magnetic fields arising from normal fields on the boundaries that closely approximated the actual normal-field boundary conditions before and after the cancellation phase. We found that the magnetic energy for these potential fields went from 0.75 before the cancellation to 0.67 after the cancellation, a drop of just over $10 \%$, while the actual energy in the volume fell from 0.77 before the cancellation to 0.73 after the cancellation, a drop of $5 \%$. Consequently, the cancellation led to an increase of $\sim 5 \%$ in the free magnetic energy in the volume.

Evidently, however, our final configurations are stable equilibria, showing no sign whatsoever of imminent eruption. We note that an eruption could not occur without first opening the uniform background field, as well as the overlying reconnected fields, which are the least sheared (and therefore the most stable) in the configuration. If the overlying field were quadrupolar, however, the free energy added by the cancellation might cause the newly created long and low-lying field lines to rise, driving reconnection between the overlying closed fields and neighboring flux systems, leading to a breakout-type eruption (Antiochos et al. 1999).

This work was supported by NASA, Berkeley's AFOSRfunded Solar-MURI program, ONR, and generous grants of computer time from the DOD High Performance Computing Modernization Program at the ERDC MSRC.
Amari, T., Luciani, J. F., Mikic, Z., \& Linker, J. 1999, ApJ, 518, L57 Antiochos, S. K., Dahlburg, R. B., \& Klimchuk, J. A. 1994, ApJ, 420, L41 Antiochos, S. K., DeVore, C. R., \& Klimchuk, J. A. 1999, ApJ, 510, 485 Aulanier, G., DeVore, C. R., \& Antiochos, S. K. 2002, ApJ, 567, L97 Berger, M. A., \& Field, G. B. 1984, J. Fluid Mech., 147, 133

\section{REFERENCES}

Bommier, V., \& Leroy, J. L. 1998, in IAU Colloq. 167, New Perspectives on

Solar Prominences (ASP Conf. Ser. 150; San Francisco: ASP), 434 Chae, J. 2003, ApJ, 584, 1084

Chae, J., Moon, Y., \& Park, S. 2003, J. Korean Astron. Soc., 36, 13 Chae, J., Moon, Y., \& Pevtsov, A. A. 2004, ApJ, 602, L65 
Chae, J., Moon, Y., Wang, H., \& Yun, H. S. 2002, Sol. Phys., 207, 73

Chae, J., Wang, H., Qiu, J., Goode, P. R., Strous, L., \& Yun, H. S. 2001, ApJ, 560,476

Démoulin, P. 1998, in IAU Colloq. 167, New Perspectives on Solar Prominences (ASP Conf. Ser. 150; San Francisco: ASP), 78

DeVore, C. R. 1991, J. Comput. Phys., 92, 142

DeVore, C. R., \& Antiochos, S. K. 2000, ApJ, 539, 954

Forbes, T. G. 2000, J. Geophys. Res., 105, 23153

Galsgaard, K., \& Longbottom, A. W. 1999, ApJ, 510, 444

Gosling, J. T. 1993, J. Geophys. Res., 98, 18937

Hale, G. E., Ellerman, F., Nicholson, S. B., \& Joy, A. H. 1919, ApJ, 49, 153

Harvey, K. L., Jones, H. P., Schrijver, C. J., \& Penn, M. J. 1999, Sol. Phys., 190,35

Karpen, J. T., Antiochos, S. K., Hohensee, M., Klimchuk, J. A., \& MacNeice, P. J. 2001, ApJ, 553, L85

Kippenhahn, R., \& Schlüter, A. 1957, Z. Astrophys., 43, 36

Kuperus, M., \& Raadu, M. A. 1974, A\&A, 31, 189

Leka, K. D., Canfield, R. C., McClymont, A. N., \& Van Driel Gesztelyi, L. 1996, ApJ, 462, 547

Linker, J. A., Lionello, R., Mikić, Z., \& Amari, T. 2001, J. Geophys. Res., 106, 25165

Lionello, R., Mikić, Z., Linker, J. A., \& Amari, T. 2002, ApJ, 581, 718

Lites, B. W. 2005, ApJ, 622, 1275

Mackay, D. H., \& Gaizauskas, V. 2003, Sol. Phys., 216, 121

Mackay, D. H., \& van Ballegooijen, A. A. 2001, ApJ, 560, 445

2005, ApJ, 621, L77
MacNeice, P., Olson, K. M., Mobarry, C., De Fainchtein, R., \& Packer, C. 2000, Comput. Phys. Commun., 126, 330

Magara, T., \& Longcope, D. W. 2001, ApJ, 559, L55

Martens, P. C., \& Zwaan, C. 2001, ApJ, 558, 872

Martin, S. F. 1998, Sol. Phys., 182, 107

Martin, S. F., Billamoria, R., \& Tracadas, P. W. 1994, in Solar Surface Magnetism, ed. R.J. Rutten \& C. J. Schrijver (NATO ASI Ser. C; Dordrecht: Kluwer), 303

Martin, S. F., \& McAllister, A. H. 1996, in IAU Colloq. 153, Magnetodynamic Phenomena in the Solar Atmosphere: Prototypes of Stellar Magnetic Activity, ed. Y. Uchida, T. Kosugi, \& H. S. Hudson (Dordrecht: Kluwer), 497 Pevtsov, A. A. 2002 in Multi-Wavelength Observations of Coronal Structure and Dynamics, ed. P. C. H. Martens \& D. Cauffman (Amsterdam: Elsevier), 125

Pevtsov, A. A., Canfield, R. C., \& Metcalf, T. R. 1995, ApJ, 440, L109

Pevtsov, A. A., Balasubramaniam, K. S., \& Rogers, J. W. 2003, ApJ, 595, 500 Priest, E. R. 1985, Rep. Prog. Phys., 48, 955

Priest, E. R., Hood, A. W., \& Anzer, U. 1989, ApJ, 344, 1010

Rust, D. M., \& Kumar, A. 1994, Sol. Phys., 155, 69

Tang, F. 1987, Sol. Phys., 107, 233

van Ballegooijen, A. A., \& Martens, P. C. H. 1989, ApJ, 343, 971

van Driel-Gesztelyi, L., Malherbe, J.-M., \& Démoulin, P. 2000, A\&A, 364, 845

Welsch, B. T., Fisher, G., \& Abbett, W. 2004, ApJ, 610, 1148

Zirker, J. B., Martin, S. F., Harvey, K., \& Gaizauskas, V. 1997, Sol. Phys., 175, 27 